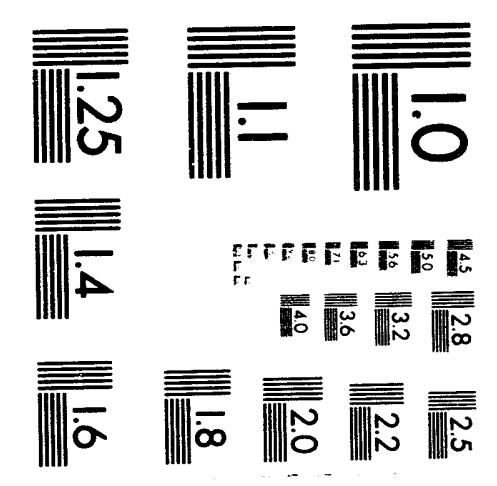



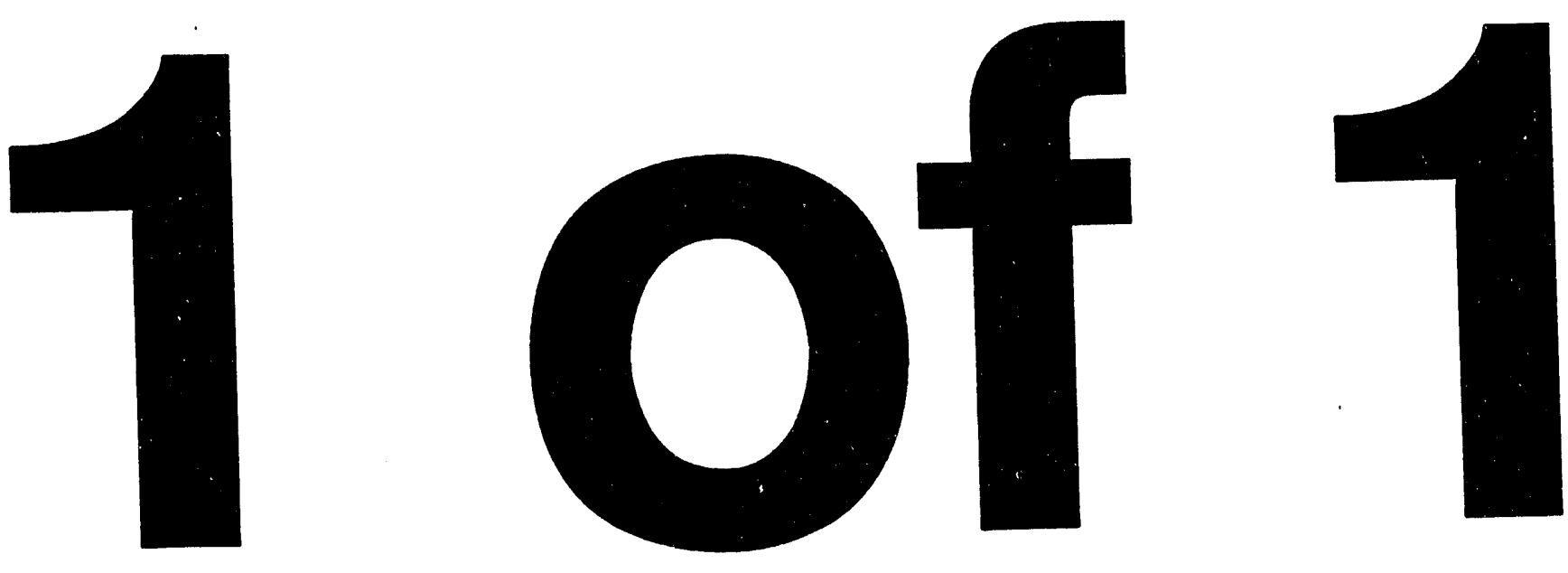


\section{SCATTERING THEORY FOR THE QUANTUM ENVELOPE OF A CLASSICAL SYSTEM}

\section{E.C.G. Sudarshan}

Center for Particle Physics, Department of Physics University of Texas, Austin, TX 78712

\section{ABSTRACT}

Classical dynamics, reformulated in terms of its quantum envelope is studied for the stationary states of the interacting system. The dynamical variable of "elapsed time" plays a crucial role in this study. It is shown that the perturbation series for the elapsed time can be summed in various simple cases even when standard perturbation series diverge. For the special class of systems where the interactions fall off sufficiently fast at infinity one could define "in" and "out" states; and consequently the wave matrices and scattering matrices. The scattering phase shifts bear a simple relation to the time delay in scattering.
${ }^{1}$ International Seminar in Honor of Professor Iwo Bialyniki-Birula, Warsaw, Poland.

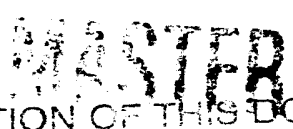

\section{Introduction}

Classical dynamics admits of an algebra of dynamical variables which are realized as functions of canonical variables. ${ }^{1} \mathrm{~A}$ Lie algebra is defined over these by Poisson brackets. ${ }^{2}$ The time evolution is governed by a Hamiltonian $h(p, q ; t)$ with the equations of motion:

$$
\dot{A}(q, p, t)=\{A, h\}_{P} . b .+\frac{\partial}{\partial t} A(q, p, t) .
$$

For small times this operator may be integrated to obtain $A\left(q^{(t)}, p^{t} ; t\right)$ as a function of $q, p, t$ and the initial value $A\left(q, p ; t_{0}\right)$. But in the limit of large times there may not be a suitable limit to the quantity as a function of time. More important is the fact that for many systems the behavior of the trajectory may be unstable in these cases of deterministic chaos; the trajectories lose any practical significance and so we are led to use a phase space density description. In all these cases, the canonical variables $q, p$ one takes to be real; and so are the energies real.

When interactions are considered for unbounded motion, we have scattering; and in scattering, with initial uniform motion with varying impact parameters, the trajectories appear to be diverging from the scattering center. This is reminiscent of the "in" states of scattering in quantum theory. The Somimerfeld outgoing wave boundary conditions ${ }^{3}$ adapted to quantum theory correspond to the energy acquiring an infinitesimal imaginary part. ${ }^{4}$ So use of complex energy states in classical wave motion is quite natural and for generic classical mechanics may not be irrelevant.

Another case where complex energies with finite imaginary parts are relevant in quantum theory is when one studies metastable states with finite lifetimes. Such resonances arise in classical mechanics also, and in particular in the context of the Poincaré catastrophe of perturbation theory. In quantum 
theory, the dynamical information of the lifetime of a metastable state (the width of a resonance) can be converted into spectral information in a reformulation of quantum theory in terms of generalized dual spaces. It is interesting to see if this can be done in classical mechanics also.

Even more generally one can ask whether a formulation of scattering theory can be carried out for classical mechanics. This would involve not only scattering cross sections but also the analogues of the Möller wave matrix with the unitarity reflecting the completeness of the free states and of the interacting states; as well as the notion of the isospectral comparison Hamiltonian.

But to bring about such a formulation we must realize classical mechanics as a vector space theory. This can be (and has been) done. ${ }^{7}$ The state vectors must realize the canonical variables $q, p$ as well as the elementary vector fields $-i \frac{\partial}{\partial q},-i \frac{\partial}{\partial p}$. This is a system of $2 N$ canonical degrees of freedom $\left(q,+i \frac{\partial}{\partial q}, p,-i \frac{\partial}{\partial p}\right)$ considered as a quantum system called the quantum envelope of the classical system. The variables $\left(-i \frac{\partial}{\partial q},-i \frac{\partial}{\partial p}\right)$ are considered non-observable. The vector space may be realized in the Schrödinger picture by $L_{2}(-\infty, \infty)$ functions of $q, p$ with a scalar product

$$
\iint \psi^{\dagger}(q, p) \psi_{2}(q, p) d q d p \equiv\left(\psi_{1}, \psi_{2}\right) \equiv\left(\psi_{1}^{\dagger} \psi_{2}\right)
$$

Expectation values of a dynamical variable $A(q, p)$ is given by

$$
\begin{aligned}
\langle A(q, p)\rangle_{\psi} & =\frac{\int \psi^{\dagger}(q, p) A(q, p) \psi(q, p) d q d p}{\int \psi^{\dagger}(q, p) \psi(q, p) d q d p} \\
& =\left(\psi^{\dagger} A \psi\right) / \psi^{\dagger} \psi \equiv(\psi, A \psi) /(\psi, \psi)
\end{aligned}
$$

Just as in quantum mechanics, it may be to our advantage to use nonnormalizable ideal eigenvectors in certain cases. This is particularly true of states of definite energy in the continuous spectrum when one encounters rigged spaces; but more generally one deals with dual spaces. ${ }^{6}$
Much of the physical interpretation of quantum mechanics include expectation values and transition matrix elements, but many of the generic properties depend on the spectral properties of the operators. In previous studies we have shown that the notion of the spectrum (even of a self-adjoint operator) is not unique and can be generalized when the vector space is "analytically continued." This is not an inconvenience but an opportunity to encode additional dynamical information in the modified spectrum. The first systematic study of such modified spectra was undertaken in the study of all unitary representations of noncompact groups by the method of Master Analytic Representations. ${ }^{9}$ Such modified spectra reappeared in the study of solvable models containing metastable excitations within the context of generalized quantum mechanics. ${ }^{10}$

It is natural to enquire whether these results obtain in the vector space treatment of classical mechanics of interacting particles. This is implemented in this paper.

\section{Classical Dynamics Embedded in a Vector Space}

Define a vector space $\mathcal{H}$ over the field of complex numbers of complex valued functions of a set of classical phase variables $q, p$ with the $L^{2}$ norm

$$
(\psi, \psi)=\int_{-\infty}^{\infty} d q \int_{-\infty}^{\infty} d p \psi^{*}(q, p) \psi(q, p)
$$

We can define the unbounded operators $Q, P$ by

$$
\begin{aligned}
& (Q \psi)(q, p)=q \psi(q, p) \\
& (P \psi)(q, p)=p \psi(q, p)
\end{aligned}
$$


and their canonical conjugates $K, X$ by

$$
\begin{aligned}
& (K \psi)(q, p)=+i \frac{\partial}{\partial q} \psi(q, p) \\
& (X \psi)(q, p)=-i \frac{\partial}{\partial p} \psi(q, p)
\end{aligned}
$$

A dense subset of vectors in $\mathcal{H}$ are realized by square integrable boundary values of functions analytic in a domain $\mathcal{D}$. They form a vector space $\mathcal{G}$. The complex conjugates $\psi^{*}(q, p)$ may be thought of as the boundary value of $\psi^{*}\left(q^{*}, p^{*}\right)$ and analytic in the same domain $\mathcal{D}$ and the same vector space $\mathcal{G}$. Within the domain of analyticity

$$
\left(\psi_{1}, \psi_{2}\right) \equiv \int_{\Gamma_{1}} \int_{\Gamma_{2}} \psi_{1}^{*}\left(q^{*}, p^{*}\right) \psi_{2}(q, p) d q d p
$$

with $q, p$ complex variables along contour $\left\{\Gamma_{1}, \Gamma_{2}\right\}$.

With the scalar product defined for normalizable vectors $\{Q, P, K, X\}$ are all hermitian. All observables, however, are functions only of $\{Q, P\}$.

The Hamiltonian evolution is given by the equation of motion

$$
\begin{aligned}
i \dot{A}(q, p, t) & =i \frac{\partial A}{\partial t}(q, p, t)+i\{A, H\}_{P . b} . \\
& \equiv i \frac{\partial A}{\partial t}+\left[\mathcal{L}_{H}, A\right]
\end{aligned}
$$

where the Liouvillian $\mathcal{L}_{H}$ is linear in $\{K, X\}$

$$
\begin{aligned}
\mathcal{L}_{H} & =\frac{\partial H}{\partial p} K+\frac{\partial H}{\partial q} X \\
& \equiv i \frac{\partial H}{\partial p} \frac{\partial}{\partial q}-i \frac{\partial H}{\partial q} \frac{\partial}{\partial p}
\end{aligned}
$$

and is hermitian but is not an observable! The time evolution is given by the Schrödinger picture equations of motion

$$
i \frac{\partial}{\partial t} \mho(q, p, t)=\left(\mathcal{L}_{H} \psi\right)(q, p, t) .
$$

and hence

$$
\psi(q, p, t)=\left(\exp \left(-i \mathcal{L}_{H} t\right) \psi\right)(q . p, 0)
$$

if $\mathcal{L}_{H}$ is not explicitly time dependent. Otherwise it is given by

$$
\psi(q, p, t)=\left(\exp \left(-i \int_{0}^{t} d t^{\prime} \mathcal{L}_{H}\left(t^{\prime}\right)\right)_{+} \psi\right)(q, p, 0)
$$

where the + subscript refers to the time ordering of $\mathcal{L}_{H}$ at different times in the integral. Since $\mathcal{L}_{H}$ is hermitian, the time-ordered exponential is unitary and the norm of the state vector is preserved.

A classical system with $N$ degrees of freedom has a quantum envelope with $2 N$ canonical pairs: for the special case of one degree of freedom we see that the quantum envelope has two degrees of freedom. Therefore there should be three operators that commute with the Liouvillian. One of them is the Hamiltonian which is an observable.

$$
\left[\mathcal{L}_{H}, H\right]=0
$$

We may choose two other operators which commute with $\mathcal{L}_{H}$ but we expect that neither of them is observable.

As far as the observables are concerned, the phase of the "Schrödinger" wavefunction $\psi(q, p)$ is irrelevant. ${ }^{7}$ Both $\psi(q, p)$ and $e^{i \theta(q, p)} \psi(q, p)$ are equivalent as far as observables are concerned since observables depend only on the density

$$
\rho(q, p)=\psi(q, p) \psi^{*}(q, p)
$$

However, for any state $\psi(q, p)$ there exists a phase $\theta(q, p)$ such that $e^{i \theta(q, p)} \psi(q, p)$ is orthogonal to $\psi(q, p)$ provided $\psi(q, p)$ is square integrable. Hence, while the phase is unobservable, the states are different as far as the vector spaces $\mathcal{H}, \mathcal{G}$ are concerned. Under the gauge transformation

$$
\psi(q, p) \rightarrow e^{i \theta(q, p)} \psi(q, p)
$$


all the observables are gauge invariant but the Liouvillian and other operators that depend on $K$ and $X$ undergo the gauge transformations ${ }^{7,8}$

$$
\begin{aligned}
\mathcal{L}_{H} & \rightarrow \mathcal{L}_{H}+\frac{\partial H}{\partial q} \frac{\partial \theta}{\partial p}-\frac{\partial H}{\partial q} \frac{\partial \theta}{\partial q} \\
K & \rightarrow K-\frac{\partial \theta}{\partial q} \quad ; X \rightarrow X+\frac{\partial \theta}{\partial p}
\end{aligned}
$$

\section{Stationary States of the Quantum Enve- lope}

Let us now consider the stationary wave functions of the quantum envelope of a free classical system with one degree of freedom with Hamiltonian

$$
H=H_{0}=\frac{p^{2}}{2}
$$

(We choose the mass to be unity and replace $q$ by $x$.) The Hamiltonian operator of the quantum envelope is

$$
\mathcal{L}=\mathcal{L}_{0}=i p \frac{\partial}{\partial x}=P K
$$

with commuting operators (we replace $q$ by $x$ ):

$$
\mathcal{M}=P^{-2} Q K-P^{-1} X=\frac{i x}{p^{2}} \frac{\partial}{\partial x}+\frac{i}{p} \frac{\partial}{\partial p}
$$

and

$$
K=i \frac{\partial}{\partial x}
$$

We recognize that

$$
\left(\frac{i x}{p^{2}} \frac{\partial}{\partial x}+\frac{i}{p} \frac{\partial}{\partial p}, \frac{p^{2}}{2}\right),\left(i p \frac{\partial}{\partial x}, \frac{x}{p}\right)
$$

are canonical pairs of dynamical variables of the quantum envelope. The quantities $\frac{p^{2}}{2}$ and $\frac{x}{p}$ have the physical significance of energy and "elapsed time." Of the canonical pairs, we can look for eigenfunctions for one each.
The states of the classical sjrstem with a constant energy (and even with constant momentum) are not stationary since the position changes linearly with time proportioned to the momentum! A stationary state should treat all positions equally. So we go to the quantum envelope and construct the eigenstates of the Liouvillian:

$$
\mathcal{L}_{0} \psi(x, p)=\lambda \psi(x, p)
$$

with the solution

$$
\psi(x, p)=\exp (-i \lambda x / p)
$$

which is also an eigenstate of $Q K-P X$ with eigenvalue 0 .

$$
\mathcal{M}_{0} \psi(x, p)=i\left(\frac{x}{p^{2}} \frac{\partial}{\partial x}+\frac{1}{p} \frac{\partial}{\partial p}\right) \psi(x, p)=0 \text {. }
$$

We may rewrite the wavefunction in the form

$$
\psi_{\lambda}(x, p)=\exp \left(-i \lambda \tau_{0}(x, p)\right)
$$

with

$$
\left[\mathcal{L}_{0}, \tau_{0}(x, p)\right]=\left\{H_{0}, \tau_{0}(x, p)\right\}_{P . b .}=1
$$

We also note that the pseudodensity matrices formed from any bilinear in the wavefunctions

$$
\psi_{\lambda_{1}}(x, p) \psi_{\lambda_{2}}^{*}(x, p)=R_{\lambda_{1} \lambda_{2}}(x, p)
$$

obey the commutations

$$
\begin{aligned}
{\left[\mathcal{L}_{0}, R_{\lambda_{1} \lambda_{2}}\right] } & =\left(\lambda_{1}-\lambda_{2}\right) R_{\lambda_{1} \lambda_{2}} \\
{\left[\mathcal{M}_{0}, R_{\lambda_{1} \lambda_{2}}\right] } & =0 \\
{\left[\frac{p^{2}}{2}, R_{\lambda_{1} \lambda_{2}}\right] } & =0 .
\end{aligned}
$$

On the other hand, for

$$
\begin{aligned}
\phi_{\mu}(x, p) & =e^{-i \mu p^{2} / 2} \\
\mathcal{L}_{0} \phi_{\mu}=0 & , \quad \mathcal{M}_{0} \phi_{\mu}=\mu \phi_{\mu}
\end{aligned}
$$


Hence

$$
\Psi_{\lambda, \mu}(x, p)=\exp \left(-i \lambda \tau_{0}(x, p)-i \mu p^{2} / 2\right)
$$

satisfies

$$
\begin{aligned}
\mathcal{L}_{0} \Psi & =\lambda \Psi \\
\mathcal{M}_{0} \Psi & =\mu \Psi
\end{aligned}
$$

and is thus a simultaneous eigenfunction. For each energy we have a phase proportional to it; and for each position we have a phase proportional to the elapsed time $\tau_{0}$. Since both these phases are not measurable, the density matrix may be replaced by a phase density independent of position; that way alone we can assure that it is stationary. Here we obtain a pure state realized by the vector $\psi_{\lambda}$ or $\Psi_{\lambda \mu}$. For some purposes it is more appropriate to consider an eigenstate of $\tau$ and $H_{0}$ :

$$
\Psi_{\lambda, E}=\delta(p-\sqrt{2 E}) e^{-i \lambda r_{0}(x, p)}
$$

Here also, as far as the observables are concerned, this state is equivalent to a space-independent phase space density.

If the origin is shifted by $a,\left(\tau_{0} x, p\right)$ changes:

$$
\tau_{0}(x, p) \rightarrow \tau_{0}(x, p)+\frac{a}{p}
$$

which is also canonically conjugate to $i p \frac{\partial}{\partial x}$

For a classical system with $N$ degrees of freedom and a Hamiltonian

$$
H_{0}=H_{1}+H_{2}+\cdots+H_{N}, H_{r}=\frac{1}{2} p_{r}^{2}
$$

we could define $N$ elapsed time variables

$$
\tau_{r}(x, p)=\frac{x_{r}}{p_{r}}
$$

All these have the same value $\tau_{0}(x, p)$

$$
\tau_{0}(x, p)=\boldsymbol{x} \cdot \boldsymbol{p} / \boldsymbol{p} \cdot \boldsymbol{p}
$$

This equality is equivalent to the vanishing of the angular momentum

$$
x_{r} p_{s}-x_{s} p_{r}=0
$$

Not all these relations are independent here; the relations $r=1$, for all values of $s$ implies the rest. If the origin is shifted

$$
x \rightarrow x+a, \tau_{r} \rightarrow \tau_{r}+\frac{a_{r}}{p_{r}}
$$

then

$$
x_{r} p_{s}-x_{s} p_{r} \rightarrow x_{r} p_{s}-x_{s} p_{r}+a_{r} p_{s}-a_{s} p_{r}
$$

So the angular momenta are not zero but constants.

In this case, a proper choice of canonical pairs is:

$$
\begin{aligned}
& \left(i \boldsymbol{p} \cdot \frac{\partial}{\partial \boldsymbol{x}}, \tau_{0}(x, p)\right) \\
& \left(x_{1} \frac{\partial}{\partial x}-x_{s} \frac{\partial}{\partial x_{1}}+p_{1} \frac{\partial}{\partial p_{s}}-p_{s} \frac{\partial}{\partial p_{1}}, \tan ^{-1} \frac{x_{s}}{x_{1}}\right) \\
& \left(i\left(\boldsymbol{p} \cdot \frac{\partial}{\partial \boldsymbol{p}}+\boldsymbol{x} \cdot \frac{\partial}{\partial \boldsymbol{x}}+\frac{\boldsymbol{x} \cdot \boldsymbol{p}}{p^{2}} \cdot \frac{\boldsymbol{p} \cdot \frac{\partial}{\partial \boldsymbol{x}}}{p^{2}}\right), \boldsymbol{p}^{2} / 2\right)
\end{aligned}
$$

\section{Elapsed Time of Interacting Systems}

For an interacting system with one degree of freedom the classical Hamiltonian is

$$
H=\frac{p^{2}}{2}+V(x)
$$

The Hamiltonian operator for the quantum envelope is

$$
\mathcal{L}=i p \frac{\partial}{\partial x}-\frac{i \partial V}{\partial x} \frac{\partial}{\partial p} \equiv \mathcal{L}_{0}+i f(x) \frac{\partial}{\partial p}
$$


The elapsed time variable $\tau(x, p)$ conjugate to $\mathcal{L}$ satisfies the linear differential equation

$$
p \frac{\partial \tau}{\partial x}+f(x) \frac{\partial \tau}{\partial p}=1
$$

or

$$
\frac{\partial \tau}{\partial x}=\frac{1}{p}-\frac{1}{p} f(x) \frac{\partial \tau}{\partial p}
$$

It has the formal solution

$$
\tau(x p)=\frac{x}{p}-\frac{1}{p} \int^{x} f(y) \frac{\partial \tau}{\partial p}(p, y) d y=\tau_{0}(x, p)-\frac{1}{p} \int^{x} f(y) \frac{\partial \tau}{\partial p} d y
$$

The perturbative solution is

$$
\tau(x, p)=\sum_{0}^{\infty}\left((-)^{n} \frac{1}{p} \int^{x} d y f\left(y_{1}\right) \frac{\partial}{\partial p} \frac{1}{p} \int^{y_{1}} d y_{2} f\left(y_{2}\right) \frac{\partial}{\partial p} \cdots\right) \frac{y_{n}}{p}
$$

For the case of no force, $V(x)=0, \tau(x, p)$ reduces to $\tau_{0}(x, p)$. For the case of constant force

$$
f(x)=a
$$

this has a simple form:

$$
\begin{aligned}
\tau(x, p) & =\frac{x}{p}+\sum_{1}^{\infty}(-)^{n}\left(\frac{a}{p}\right)^{n} \int^{x} d y_{1} \int^{y_{1}} d y_{2} \cdots \int d y_{n}\left(\frac{\partial}{\partial p}\right)^{n} \frac{y_{n}}{p} \\
& =\sum_{0}^{\infty} \frac{a^{n} x^{n+1}(2 n-1) ! !}{p^{2 n+1} n !}
\end{aligned}
$$

But this series can be seen to be the expansion of

$$
\left\{\left(1+\frac{2 a x}{p^{2}}\right)^{\frac{1}{2}}-1\right\} / 2 a=\tau
$$

with

$$
p \tau+\frac{1}{2} a \tau^{2}=x
$$

Thus $\tau(x, p)$ is, in fact, the elapsed time for motion under constant acceleration. Similarly, for harmonic motion

$$
f(x)=-k x
$$

the series solution for $\tau(x, p)$ gives

$$
\begin{aligned}
\tau(x, p) & =\frac{x}{p}+\sum_{1}^{\infty}\left(-k^{n} \frac{\partial^{n}}{\partial p^{n}} \int^{x} d y_{1} y_{1} \int^{y_{1}} d y_{2} y_{2} \cdots \int^{y_{n-1}} d y_{n} y_{n}\right) \frac{x}{p} \\
& =\frac{k x}{p}-\frac{1}{3}\left(\frac{k x}{p}\right)^{3}+\frac{1}{5}\left(\frac{k x}{p}\right)^{5}++\sum_{0}^{\infty} \frac{(-)^{n}}{2 n+1}\left(\frac{k x}{p}\right)^{2 n+1}=\tan ^{-1}\left(\frac{k x}{p}\right.
\end{aligned}
$$

In the generic case we define $\tau(x, p)$ as a solution of the partial differential equation

$$
p \frac{\partial \tau}{\partial x}+f(x) \frac{\partial \tau}{\partial p}=1
$$

The arbitrariness in this function is by a function of the Hamiltonian

$$
\frac{p^{2}}{p}+V(x)=\frac{p^{2}}{p}+\int^{x} f(y) d y .
$$

Then

$$
\mathcal{L}=i p \frac{\partial}{\partial x}+i f(x) \frac{\partial}{\partial p}, \tau(x, p)
$$

constitute a canonical pair. Another conjugate pair commuting with $\mathcal{L}, \tau$ is

$$
\mathcal{M}=i \frac{\partial \tau}{\partial p} \frac{\partial}{\partial x}+i \frac{\partial \tau}{\partial x} \frac{\partial}{\partial p}, \frac{p^{2}}{2}-\int^{x} v(y) d y
$$

The eigenfunctions of the quantum envelope are given by:

$$
\Psi_{\lambda, E}^{( \pm)}=\frac{1}{2 \pi} \delta\left(p^{2}-2 E+2 V(x)\right) e^{-i \lambda \tau(x, p)}, p= \pm \sqrt{2 E}
$$

Note that $\mathcal{L}$ commutes with the delta function "normalization factor."

If we have more than one degree of freedom for the classical system, there are degeneracies among the functions $\Psi_{\lambda, E}$ which are eigenfunctions of the Liouville operator. They may be chosen from among the invariants of motion. The states with \pm denoting the sign of $p$ for a given $E$,

$$
\Psi_{\lambda, E}^{ \pm}(x, p)=\frac{\sqrt{p}}{\sqrt{2 \pi}} \delta\left(\frac{p^{2}}{2} E+V(x)\right)\left(\frac{p^{2}}{2}+V-E\right) e^{-i \lambda \tau(x, p)}
$$


constitute an orthonormal ideal set:

$$
\begin{aligned}
& \left(\Psi_{\lambda E}^{( \pm)}, \Psi_{\lambda^{\prime} E^{\prime}}^{ \pm}\right)=\frac{1}{2 \pi} \int d x \int p d p \Psi_{\lambda E}^{*}(x, p) \Psi_{\lambda^{\prime} E^{\prime}}(x, p) \\
= & \frac{1}{2 \pi} \int d \tau \int d\left(\frac{p^{2}}{2}+V(x)\right) \delta\left(E-\frac{p^{2}}{2}-V(x)\right) \delta\left(E^{\prime}-\frac{p^{2}}{2} V(x)\right) \\
= & \delta\left(\lambda-\lambda^{\prime}\right) \delta\left(E-E^{\prime}\right) \exp \left\{i\left(\lambda-\lambda^{\prime}\right) \tau(x, p)\right\} \\
& \left(\Psi_{\lambda E}^{( \pm)}, \Psi_{\lambda^{\prime} E^{\prime}}^{(f)}\right)=0 .
\end{aligned}
$$

They are also complete:

$$
\begin{gathered}
\sum_{ \pm} \iint \Psi_{\lambda E}^{( \pm)}(x, p) \Psi_{\lambda E}^{( \pm)}\left(x^{\prime} p^{\prime}\right) d E d \lambda=\sum_{ \pm} \frac{1}{2 \pi} \iint \delta\left(\frac{p^{2}}{2} \mp(E-V)\right) \\
\delta\left(\frac{p^{\prime 2}}{2}\right)-\left(E-V\left(x^{\prime}\right)\right) e^{i \lambda\left(\tau\left(x^{\prime}, p^{\prime}\right)-\tau(x, p)\right)} d E d \lambda \\
=\delta\left(\tau(x, p)-\tau\left(x^{\prime} p^{\prime}\right)\right) \delta\left(\frac{p^{2}}{2}+V(x)-\frac{p^{\prime 2}}{2}-V\left(x^{\prime}\right)\right) \delta( \pm, \pm) .(4.20)
\end{gathered}
$$

Hence, any wavefunction can be expanded in this ideal basis. Equally well, for the case of $V(x)=0$, we obtain $\Psi_{\lambda, E}$ to be ideal orthonormal and complete.

Consider the integro-differential operator

$$
W^{-1}=\left\{1-\left(i p \frac{\partial}{\partial x}\right)^{-1} f(x) i \frac{\partial}{\partial p}\right\}
$$

and its action on the Hamiltonian:

$$
\begin{aligned}
\{1 & \left.+\left(i p \frac{\partial}{\partial x}\right)^{-1} f(x) i \frac{\partial}{\partial p}\right\}\left\{\frac{p^{2}}{2}+V(x)\right\} \\
& =\frac{p^{2}}{2}+V(x)-\left(i p \frac{\partial}{\partial x}\right)^{-1} f(x) i \frac{\partial}{\partial p}\left(\frac{p^{2}}{2}\right)=\frac{p^{2}}{2}+V(x)-\left(\frac{\partial}{\partial x}\right)^{-1} f(x)=\frac{p^{2}}{2} .
\end{aligned}
$$

Hence

$$
W \frac{p^{2}}{2}=\left(\frac{p^{2}}{2}+V(x)\right)
$$

In addition we also saw that

$$
W \tau_{0}(x, p)=W \frac{x}{p}=\tau(x, p)
$$

The generic solutions $\Psi_{\lambda, E}(x, p)$ are given by

$$
\begin{aligned}
\Psi_{\lambda, E}(x, p) & =\sqrt{\frac{|p|}{2 \pi}} \delta\left(\frac{p^{2}}{2}-E+V(x)\right) e^{i \lambda \tau(x, p)} \\
& =\sqrt{\frac{|p|}{2 \pi}} \delta\left(W \frac{p^{2}}{2}-E\right) e^{i \lambda\left(W \tau_{0}(x, p)\right)}
\end{aligned}
$$

While the free particle states are complete, for attractive potentials there are bound states which do not obtain in this fashion. Such states may be obtained from among the free particle solutions with real values of $\tau_{0}(x, p)=\frac{x}{p}$ and negative values of $E$. The bound states of the Hamiltonian corresponding to negative values of $E$ constitute a continuum. Formally $\delta\left(\frac{1}{2} p^{2}+|E|\right) e^{-i \lambda \frac{\mid x}{\sqrt{2|E|}}}$ is a solution with eigenvalue $\lambda$ for $\mathcal{L}_{0}=i p \frac{\partial}{\partial x}$ and energy $-|E|$. The transformed solution will be

$$
\delta\left(\frac{1}{2} p^{2}+V(x)+|E|\right) e^{-i \lambda \tau(x p)}
$$

with both $x, p$ being real.

For the class of Hamiltonians with a local potential

$$
H(x, p)=\frac{1}{2} p^{2}+V(x)
$$

the elapsed time $\tau(x, p)$ can be defined by the integral

$$
\tau(x, p)=\int_{x_{1}}^{x} \frac{d y}{\sqrt{2 \mu-2 V(y)}}
$$

with $\mu$ being replaced after the integration by $H(x, p)$; or equally well left unchanged, it being understood that the expression is valid for the specific total energy $\mu$. We verify that $\tau(x, p)$ reduces to $\frac{x}{p}$ for $V(x)=0$; and, that, in all cases

$$
\begin{aligned}
\{\tau(x, p), H(x, p)\} & =\frac{\partial \tau}{\partial x} \frac{\partial H}{\partial p}=p \frac{\partial \tau}{\partial x} \\
& =p \frac{1}{\sqrt{2 \mu-2 V(x)}}=1
\end{aligned}
$$


Note that since $H(x, p)$ has vanishing Poisson bracket with $H(x, p)$, it does not matter that we have incorporated the explicit $p$ dependence of $\tau(x, p)$ into its dependence on $\mu$ which may be treated as inert when taking the Poisson bracket with $H(x, p)$.

\section{The Wave Operators in the Quantum En- velope}

Given the exact solutions $\psi$ for $H$, we could reinterpret the eigenvalue equations

$$
\begin{aligned}
& \left(H \psi_{\lambda, \mu}\right)(x, p)=\mu \psi_{\lambda ; \mu}(x, p) \\
& \left(\mathcal{L} \psi_{\lambda, \mu}\right)(x, p)=\lambda \psi_{\lambda, \mu}(x, p)
\end{aligned}
$$

to be acting on a matrix

$$
U_{x, p ; \lambda \mu} \equiv \psi_{\lambda, \mu}(x, p)
$$

which has the property:

$$
H U=\mu U ; \mathcal{L} U=\lambda U
$$

We could also write

$$
U^{\dagger} U=1
$$

expressing the (ideal) orthonormalization. If the states are complete

$$
U U^{\dagger}=1
$$

Instead of writing $U$ in the form of functions (or distributions) of $x$ and $p$ we may reexpress them in a basis made up of the free system wavefunctions $\phi_{\ell, E}(x, p)$ which are also orthonormal and complete. In this basis $\mathcal{L}$ and $H$ are diagonal with eigenvalues $\ell$ and $E$. We get

$$
\begin{aligned}
\Omega_{\ell e, \lambda \mu} & =\iint \psi_{\lambda, \mu}(x, p) \phi_{\ell E}^{*}(x, p) d x d p \\
\Omega^{\dagger} \Omega & =1-\mathcal{P}_{B} \quad \Omega \Omega^{\dagger}=1-\mathcal{P}_{B},
\end{aligned}
$$

where $P_{B}$ is the projection to the bound states of $H$.

$$
H \Omega=\Omega H_{0} \quad ; \quad \mathcal{L} \Omega=\Omega \mathcal{L}_{0} .
$$

Note that $\ell, E$ do not run over the same range as $\lambda, \mu$. The latter contains negative energy bound states; such states do not obtain for the free system. If we need full correspondence, we must, include these by augmenting the spectrum of $H_{0}$ by such negative energy states to obtain a comparison Hamiltonian $H_{C}$ which would be isospectral with $H$. When $\Omega$ is also so augmented, we have the simpler set:

$$
\begin{aligned}
\Omega^{\dagger} \Omega=1 & ; \quad \Omega \Omega^{\dagger}=1 \\
H \Omega=\Omega H_{0} & ; \quad \mathcal{L} \Omega=\Omega \mathcal{L}_{0} .
\end{aligned}
$$

This is in analogy with the situation in quantum theory where we use $H$ or $\mathcal{L}$ depending upon whether we work with state vectors or density matrices. ${ }^{6}$ Here we use both.

For most systems of interest it is not possible to carry out the computations in clased form. But we could outline the calculations for the generic case.

$$
\begin{aligned}
\psi_{\lambda, \mu}(x, p) & =\int d p p \delta\left(\frac{1}{2} p^{2}+V(x)-\mu\right) e^{i \lambda \tau(x, p)} \\
\phi_{\ell, E}(x, p) & =\int d p p \delta\left(\frac{1}{2} p^{2}-E\right) e^{-i \ell x / p}
\end{aligned}
$$

Hence

$$
\begin{aligned}
\Omega_{\ell E, \lambda \mu} & =\iint d x d p \psi_{\lambda \mu}(x, p) \phi_{\ell E}^{*}(x p) \\
& =\iint p d p d x \delta\left(\frac{p^{2}}{2}-E\right) \delta\left(\frac{p^{2}}{2}+V(x)-\mu\right) e^{i \ell \frac{x}{p}-i \lambda \tau(x, p)} \\
& =\int d x \delta(E+V(x)-\mu) \exp \left\{i \ell \frac{x}{p_{1}}-i \lambda \tau\left(x \cdot p_{1}\right)\right\} \\
& =\frac{1}{V^{\prime}(x)} \exp \left\{i \ell \frac{x_{1}}{p_{1}}-i \lambda \tau\left(x_{1}, p_{1}\right)\right\}
\end{aligned}
$$


where $x_{1}, p_{1}$ are defined by:

$$
V\left(x_{1}\right)=\mu-E, \frac{p_{1}^{2}}{2}=E
$$

Thus

$$
\Omega_{\ell E, \lambda \mu}=\frac{1}{V^{\prime}\left(x_{1}\right)} \exp \left\{i\left(\frac{\ell x_{1}}{p_{1}}-\lambda \tau\left(x_{1}, p_{1}\right)\right)\right\} .
$$

The unitarity of $\Omega$ may be verified by direct calculation from this explicit expression.

The generic expression for $\tau(x, p)$ contains an integration constant. For systems like the har:nonic oscillator the potential is present at arbitrary distances and at all stages of motion; the interactions are persistent at all stages of motion; the interactions are persistent as experienced by any trajectory; in fact there are no trajectories which go to infinity. On the other hand for potentials like $\frac{e}{\left(1+x^{2}\right)}$ the potential falls off at infinite distance and hence asymptotically the trajectories become flat. It is for such potentials that we can describe scattering. In the next section we confine our attention exclusively to Hamiltonians which admit of a scattering description.

For a system with Hamiltonian

$$
H=\frac{p^{2}}{2}+V(x)
$$

with $V(x)$ going to zero "sufficiently fast" when $|x|$ goes to infinity. In these cases we could describe trajectories which have a prescribed form "at infinity". For such Hamiltonians there are two natural choices for the elapsed time variable:

$$
\begin{aligned}
\tau_{\text {in }}(x, p) & =\underset{x_{1} \rightarrow-\infty}{L t}\left\{\int_{x_{1}}^{x} \frac{d y}{\sqrt{2 H-2 V(y)}}+\frac{x_{1}}{\sqrt{2 H}}\right\} \\
\tau_{\text {out }}(x, p) & ={ }_{x_{2} \rightarrow+\infty}^{L t}\left\{-\int_{x}^{x_{2}} \frac{d y}{\sqrt{2 H-2 V(y)}}+\frac{x_{2}}{\sqrt{2 H}}\right\} .
\end{aligned}
$$

In both these integrations $H$ is considered as a constant. Correspondingly there are two wavefunctions

$$
\begin{aligned}
& \psi_{\lambda \mu}^{\text {in }}(x, p)=p^{\frac{1}{2}} \delta\left(\left(\frac{p^{2}}{2}\right)+V(x)-\mu\right) e^{-i \lambda \tau_{\text {in }}(x, p)} \\
& \psi_{\lambda \mu}^{\text {out }}(x, p)=p^{\frac{1}{2}} \delta\left(\left(\frac{p^{2}}{2}\right)+V(x)-\mu\right) e^{-i \lambda \tau_{\text {out }}(x, p)}
\end{aligned}
$$

These are ideal orthogonal wavefunctions. The scalar product of the two is the unitary matrix:

$$
\begin{aligned}
S_{\lambda \mu, \lambda^{\prime} \mu^{\prime}} & =\iint d x d p \psi_{\lambda \mu}^{\text {in }}(x, p) \psi_{\lambda^{\prime} \mu^{\prime}}^{* \text { out }}(x, p) \\
& =\delta\left(\mu, \mu^{\prime}\right) \exp \left(i \lambda^{\prime} \tau_{\text {out }}\left(s, p_{0}\right)-i \lambda \tau_{\text {in }}\left(x, p_{0}\right)\right)
\end{aligned}
$$

If we specialize this to $\lambda=\lambda^{\prime}=1$ then

$$
S_{\mu, \mu^{\prime}}=\delta_{\mu, \mu^{\prime}} \exp \left(i \int_{-\infty}^{\infty}\left\{\frac{d y}{\sqrt{2 H-2 V(y)}}-\frac{d y}{\sqrt{2 H}}\right\}\right)=\delta_{\mu, \mu^{\prime}} S(\mu) .
$$

The phase of $S$ so constructed is the difference between the time elapsed for the potential less the time elapsed for a free particle of the same en. $\mathrm{rgy}$; this is the phase delay. Thus

$$
S(\mu)=\exp (2 i \delta(\mu))
$$

where $2 \delta(\mu)$ is the phase delay. The phase delay is computed using $H=\mu$ and covers the entire motion.

In this one dimensional case the "scattering" is not in direction (since there is only one direction!) but in the phase delay and the possibility of backscattering. To get directional scattering we should proceed to discuss problems in more spatial dimensions. 


\section{Scattering in Three Dimensions}

For three dimensional motion $\boldsymbol{x}$ and $\boldsymbol{p}$ are 3-vectors and the interacting Hamiltonian with central potentials is

$$
H=\frac{p^{2}}{2}+V(r), r=|x|
$$

In this case $H, \boldsymbol{p}$ and $\boldsymbol{x} \times \boldsymbol{p}=\boldsymbol{J}$ are conserved. For the free particle case

$$
H_{0}=\frac{p^{2}}{2}
$$

the elapsed time dynamical variable conjugate to the free Liouvillian

$$
\mathcal{L}_{0}=i p \frac{\partial}{\partial x}
$$

is given by

$$
\tau_{0}(\boldsymbol{x}, \boldsymbol{p})=\frac{\boldsymbol{p} \cdot \boldsymbol{x}}{p^{2}}+\text { const. }
$$

For the interacting Liouvillian

$$
\mathcal{L}=i p \cdot \frac{\partial}{\partial x}+f(x) \cdot \frac{\partial}{\partial p}, f=-\frac{\partial}{\partial x} V
$$

the conjugate elapsed time variable $\tau(x, p)$ satisfies the integral equation

$$
\left\{1-\left(\boldsymbol{p} \cdot \frac{\partial}{\partial \boldsymbol{x}}\right)^{-1} \boldsymbol{f}(\boldsymbol{x}) \cdot \frac{\partial}{\partial \boldsymbol{p}}\right\}^{-1}\left(\frac{\boldsymbol{p} \cdot \boldsymbol{x}}{p^{2}}\right)=\tau(\boldsymbol{x}, \boldsymbol{p}) .
$$

This equation can be solved by a perturbation series

$$
\tau(\boldsymbol{x}, \boldsymbol{p})=\sum_{n=0}^{\infty}\left\{\left(\boldsymbol{p} \cdot \frac{\partial}{\partial x}\right)^{-1} f(x) \cdot \frac{\partial}{\partial \boldsymbol{p}}\right\}^{n}\left(\frac{\boldsymbol{p} \cdot \boldsymbol{x}}{p^{2}}\right)
$$

We can choose the state as an (ideal) eigenfunction of the Hamiltonian $H$, the Liouvillian $\mathcal{L}$, the angular momentum $J$ and the maximal subset of the rotation operators $\tilde{\boldsymbol{J}}$ where

$$
\tilde{J}=i\left(\boldsymbol{x} \times \frac{\partial}{\partial \boldsymbol{x}}+\boldsymbol{p} \times \frac{\partial}{\partial \boldsymbol{p}}\right)
$$

Note that while $\tilde{J}$ commutes with $, H, \mathcal{L}$ its components do not commute; and one may choose $\tilde{J}_{3}$ and $\vec{J}^{2}$ as a complete commuting set. The angular momentum $\boldsymbol{J}$ is normal to the plane containing the orbit and is independent of the coordinate system defining the orbit; for example the two orbits in the same plane which are obtained from each other by rotation around an axis normal to the plane through the center of force have the same angular momentum. But a state with definite value of $\tilde{J}_{3}, \tilde{J}^{2}$ is defined by a superposition of such orbits for a fixed value of $\boldsymbol{J}$, with the third axis parallel to $J$. So we may choose as the constants of motion.

$$
H, \mathcal{L}, \boldsymbol{J}, \tilde{\boldsymbol{J}}^{2}, \boldsymbol{J} \cdot \tilde{\boldsymbol{J}}
$$

These states may be obtained from the states with

$$
\varphi_{\boldsymbol{k}, \boldsymbol{b}}(\boldsymbol{x}, \boldsymbol{p})=\delta(\boldsymbol{p}-\boldsymbol{k}) \delta(\boldsymbol{p} \times \boldsymbol{x}-\boldsymbol{b}) e^{-i \lambda \tau(\boldsymbol{x}, \boldsymbol{p})}
$$

by forming a linear combination with respect to all possible directions of $\boldsymbol{k}$ and $\boldsymbol{b}$, (or $\boldsymbol{k}$ and $\boldsymbol{k} \times \boldsymbol{b}$ ) with weights appropriate to the rotation group.

But in this case it is better to use $J$ and the angular directions $\theta, \phi$ of the trajectory to label the state since these are more directly related to the scattering problem. The directions are continually changing under the interaction, but under central forces the orbit is planar.

For Hamiltonians which are asymptotically free so that all the trajectories become straight lines asymptotically the scattering angle can be defined by the formula

$$
\begin{aligned}
\Theta & =2 \int_{r_{0}}^{\infty} \frac{J}{r^{2}} \frac{d r}{\sqrt{2(E-V(r))-\frac{2 J^{2}}{r^{2}}}} \\
& =2 b \int_{r_{0}}^{\infty}\left(1-\frac{V(r)}{E}-\frac{E b^{2}}{r^{2}}\right)^{-\frac{1}{2}} r^{-2} d r \\
& =2 \int_{0}^{u_{0}}\left(1-\frac{V\left(\frac{1}{u}\right)}{E}-E b^{2} u^{2}\right)^{-\frac{1}{2}} d u=F(b, E)
\end{aligned}
$$


where

$$
J=\sqrt{J^{2}}=p b
$$

and $r_{0}$ is the perihelion distance

$$
1-\frac{V\left(r_{0}\right)}{E}-\frac{E b^{2}}{r_{0}^{2}}=0 .
$$

The differential scattering cross section is

$$
\frac{d \partial}{d \Omega}=\frac{2 \pi b d b}{\sin \Theta d \Theta}=2 \pi b / \frac{\partial}{\partial b} \cos F(b, E) .
$$

These classical results can be computed in terms of the "in" and "out" wavefunctions. The "in" functions are defined in terms of the elapsed time variable

$$
\begin{aligned}
\tau_{\text {in }}(x, p)= & \underset{r_{p} \rightarrow \infty}{L t}\left[\int_{r_{\mathrm{p}}}^{r} \frac{r^{\prime}\left|d r^{\prime}\right|}{\sqrt{2\left\{r^{\prime 2}\left(E-V\left(r^{\prime}\right)\right)-J^{2}\right\}}}\right. \\
& \left.-\int_{r_{\mathrm{p}}}^{r} \frac{r^{\prime}\left|d r^{\prime}\right|}{\sqrt{2\left\{r^{\prime 2} E-J^{2}\right\}}}\right] .
\end{aligned}
$$

Further, the wavefunction is defined with respect to the 1-axis along the direction of the incident $(t \rightarrow-\infty)$ momentum and the angular momentum along the 3-axis. The state may then be labelled by the asymptotic incident momentum $k$ and impact parameter $b$. Similarly the "out" states maybe labelled by the asymptotic outgoing momentum $\boldsymbol{k}^{\prime}$ and impact parameter $\boldsymbol{b}^{\prime}$. They will be exponentially dependent on $\tau_{\text {in }}(x, p)$ and

$$
\tau_{\text {in }}(x, p)=\underset{r_{p} \rightarrow+\infty}{L t}\left[\int_{r_{p}}^{r} \frac{r^{\prime}\left|d r^{\prime}\right|}{\sqrt{2\left(E r^{\prime 2}-J\right)}}\right] .
$$

In these integrals the variable $r^{\prime}$ described the circuit $\infty$ to $r_{0}$ and $r_{0}$ to $\infty$; for the excursion from $\infty$ to $r_{0}$ we use $\left|d r^{\prime}\right|$ and for the excursion from $r_{0}$ to $\infty d r^{\prime}$ is positive.

The correlation between the asymptotic incident momentum $k$ and the asymptotic outgoing momentum $\boldsymbol{k}^{\prime}$ is the cosine of the scattering angle $2 \Theta$.

$$
\cos 2 \Theta=k, k^{\prime} / k^{2}=k \cdot k^{\prime} / 2 E .
$$

As mentioned above $\Theta$ is a functional of $V(r)$ and depends on $E$ and $J$. The total elapsed time for $E, J$ is

$$
\begin{aligned}
\tau(E, J, V(\cdot)) & =\tau_{\text {in }}(r)-\tau_{\text {out }}(r) \\
& =\tau_{\text {in }}(\infty) .
\end{aligned}
$$

This gives the relative time retardation as compared with a free particle with the same energy and angular momentum. This time delay and the scattering angle are the observables of the scattering process.

\section{Concluding Remarks}

In this paper we have continued the study of the quantum envelope of a classical system and found the complete set of eigenstates of the Liouville operator at a fixed energy. In this construction we need to construct the dynamical variable of elapsed time $\tau(x, p)$ cr ugate to the Hamiltonian. A perturbation theory to evaluate $\tau(x, p)$ is developed, it has been used to compute $\tau(x, p)$ in various cases. In the case of potentials such that for any unbounded trajectory $\tau(x, p)$ asymptotically approaches $\tau_{0}(x, p)$ the free particle value we can define the phases in a unique manner. appropriate for the "in" and "out" states. This construction enables us to recognize the "Möller wave operator" for the quantum envelope and hence the $S$-matrix. The formalism could be further developed to compute the scattering cross sections and compare it with the standard method of calculation.

The formalism developed here could be used to study resonances and the Poincaré catastrophe. These are to form the subject of a later paper.

\section{References}

1. E.T. Whitaker, "Analytical Dynamics", Dover Publications (New York 
1944).

2. E.C.G. Sudarshan and N. Mukunda, "Classical Dynamics", John Wiley (New York 1974).

3. A. Sommerfield, quoted in W. Pauli: "Electrodynamics", Academic Press (New York 1952).

4. N.F. Mott and H.S.W. Massey, "Theory of Atomic Collisions", Oxford University Press (Oxford 1965);

R.G. Newton, "Scattering Theory of Waves and Particles", Mc Graw Hill (New York 1966);

T-Y. Wu and Ohmura, "Quantum Theory of Scattering", Prentice-Hall (Englewood Cliffs, N.J. 1962); M.L. Goldberger and K.M. Watson, "Collision Theory", John Wiley (New York 1974).

5. H. Poincaré, Acta Mathematica 13, 259 (1890);

T. Petrosky and I. Prigogine, Proceedings of Nat. Acad. Sc. (US) (in press).

6. E.C.G. Sudarshan, Phys. Rev A46, 37 (1992);

E.C.G. Sudarshan and C.B. Chiu, Phys. Rev D47, 2602 (1993);

E.C.G. Sudarshan, Quantum Dynamics in Dual Spaces, DOE-ER-40757020 .

7. E.C.G. Sudarshan, Phys. Rev. D18, 4580 (1978);

T.N. Sherry and E.C.G. Sudarshan, Phys. Rev. D20, 857 (1979);

T.N. Sherry, S.R. Gautam and E.C.G. Sudarshan, Phys. Rev. D20, 3081 (1979).

8. E.C.G. Sudarshan, The Quantum Envelope of a Classical System, Proceedings of the Symposium in honor of Susumu Okubo, ed. Ashok Das,
World Scientific (1991)

9. J.G. Kuriyan, N. Mukunda and E.C.G. Sudarshan, Comm. Math Phys. 8, 204 (1968);

J. Math. Phys. 9, 12 (1968)

G. Lindbladt, Comm. Math. Phys. 48, 119 (1976).

10. E.C.G. Sudarshan, S.B. Chiu and V. Gorini, Phys. Rev. D18, 2914 (1976);

V. Gorini, G. Parravicini and ECG Sudarshan, J. Math. Phys. 21, 2205 (1980).

\section{DISCLAIMER}

This report was prepared as an account of work sponsored by an agency of the United States Government. Neither the United States Government nor any agency thereof, nor any of their employees, makes any warranty, express or implied, or assumes any legal liability or responsibility for the accuracy, completeness, or usefulness of any information, apparatus, product, or process disclosed, or represents that its use would not infringe privately owned rights. Reference herein to any specific commercial product, process, or service by trade name, trademark, manufacturer, or otherwise does not necessarily constitute or imply its endorsement, recommendation, or favoring by the United States Government or any agency thereof. The views and opinions of authors expressed herein do not necessarily state or reflect those of the United States Government or any agency thereof. 

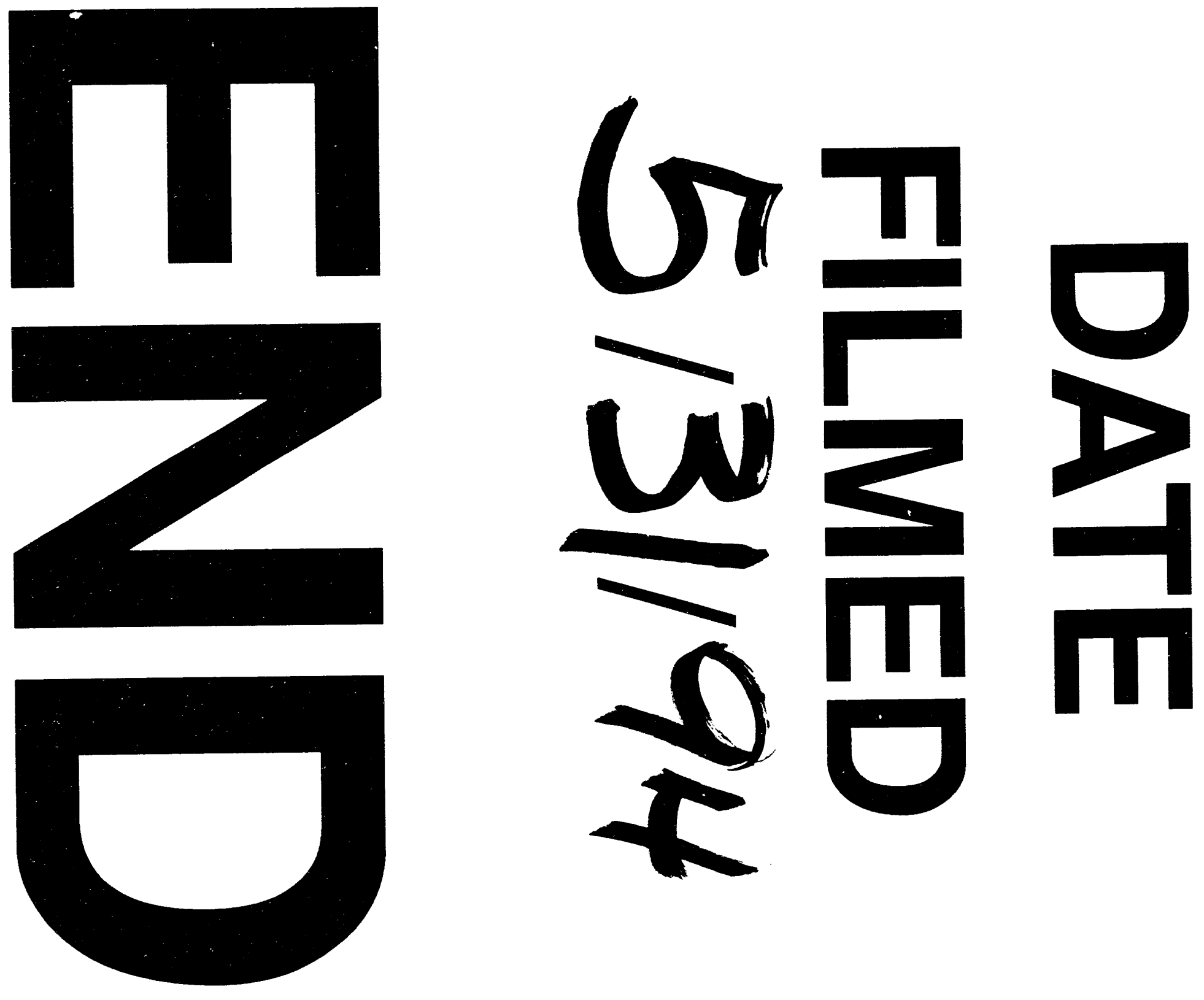


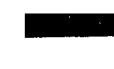

